\title{
De la educación virtual a la virtualización de procesos educativos; una transición en el contexto de las formas emergentes de cibercultura*
}

Julio Ernesto Rojas Mesa**

Linda Alejandra Leal ${ }^{* *}$

Recibido: 21 de enero de 2014 - Aprobado: 18 de marzo de 2014

\section{Resumen}

El presente artículo aborda un análisis de la relación entre la educación superior y las tecnologías de la información y la comunicación (TIC), a través de un estudio arqueológico y etnográfico en tres instituciones colombianas de educación superior: la Universidad Pedagógica Nacional (UPN), la Universidad Nacional Abierta y a Distancia (UNAD) y la Universidad Santo Tomás (USTA). El trabajo se centró en el análisis de los discursos, las prácticas y los ambientes de la educación virtual en estas instituciones.

Palabras clave: educación virtual, virtualización, perspectiva teórico/metodológica, campos emergentes, cibercultura, educación superior.

* Este artículo presenta parte de los resultados de la tesis doctoral elaborada por el doctor Julio Ernesto Rojas, Educación virtual: del discurso teórico a las prácticas pedagógicas en la educación superior colombiana. El tema ha sido continuado en la investigación: "Identificación y análisis de campos transdisciplinares emergentes", mediante la implementación de un grupo de cursos masivos abiertos y en línea (MOOC). Esta primera fase de la investigación se llevó a cabo entre febrero del 2013 y febrero del 2014, y fue financiada por la Escuela de Ciencias Sociales, Artes y Humanidades de la Universidad Nacional Abierta y a Distancia (UNAD).

** Ph.D en Teoría de la Educación y Pedagogía Social. Docente investigador de la Universidad Nacional Abierta y a Distancia (UNAD). Dirección Postal: Calle 14 Sur No. 14-23, Bogotá, Colombia. Correo electrónico: julio.rojas@unad. edu.co.

*** M.Sc. en Tecnologías de Información Aplicadas a la Educación. Docente auxiliar de la Universidad Pedagógica Nacional. Dirección Postal: Calle 14 Sur No. 14 - 23, Bogotá, Colombia. Correo electrónico: lalealu@pedagogica.edu.co. 


\title{
From virtual education to the virtualization of educational processes; a transition in the context of emerging forms of cyberculture*
}

\author{
5Julio Ernesto Rojas Mesa** \\ 6 Linda Alejandra Leal ${ }^{* * *}$
}

The present article addresses an analysis of the relationship between the higher education and the information and communication technologies ICT through an archaeological and ethnographic study in three Colombian institutions of higher education: Universidad Pedagógica Nacional -UPN, Universidad Nacional Abierta y a Distancia-UNAD, and Universidad Santo Tomás -USTA. The work was focused on the analysis of the speeches, the practices and the environments of the virtual education in these institutions.

Keywords: Virtual education, Virtualization, theoretical / methodological perspective, emerging fields, Cyberculture, higher education.

\footnotetext{
This article presents some of the results of the doctoral dissertation written by Dr. Julio Ernesto Rojas, "Virtual education: from the theoretical discourse to pedagogical practices in Colombian higher education". The theme was continued in the investigation: "Identification and analysis of emerging cross-disciplinary fields", by implementing a group of massive and open online courses (MOOC). This first phase of research was conducted between February 2013 and February 2014, and was funded by the School of Social Sciences, Arts and Humanities, of the Universidad Nacional Abierta y a Distancia -UNAD.

** Ph.D in Educational Theory and Social Education. Teaching researcher at the Universidad Nacional Abierta y a Distancia -UNAD. Address: Calle 14 Sur No. 14-23, Bogotá, Colombia. Email: julio.rojas@unad.edu.co.

*** M.Sc. Information Technology Applied to Education. Assistant professor at the Universidad Pedagógica Nacional . Address: Calle 14 Sur No. 14-23, Bogotá, Colombia. Email: lalealu@pedagogica.edu.co.
} 


\title{
De l'éducation virtuelle au virtualisation de processus éducatifs; une transition dans le contexte des formes émergentes de cyberculture*
}

\author{
Julio Ernesto Rojas Mesa** \\ Linda Alejandra Lea**
}

\section{Résumé}

Le présent article aborde une analyse de la relation entre l'éducation supérieure et les technologies de l'information et la communication TIC à travers d'une étude archéologique et ethnographique dans trois institutions colombiennes d'éducation supérieure : La Universidad Pedagógica Nacional -UPN, la Universidad Nacional Abierta y a Distancia -UNAD, et La Universidad Santo Tomas -USTA. Le travail s'est concentré sur l'analyse des discours, les pratiques et les atmosphères de l'éducation virtuelle dans ces institutions.

Mots clés: Education Virtuelle, perspective théorique -méthodologique, Virtualisation, Champs émergents, Cyberculture, Education supérieure.

Cet article présente quelques-uns des résultats de la thèse de doctorat écrite par le Dr Julio Ernesto Rojas, l'enseignement virtuel: le discours théorique à des pratiques pédagogiques dans l'enseignement supérieur colombien. Le thème a été poursuivie dans l'enquête: «Identification et analyse des champs transdisciplinaires émergents», en mettant en place un groupe de cours en ligne massives et ouvertes (Mooc). Cette première phase de la recherche a été menée entre Février 2013 et Février 2014, et a été financé par l'École des sciences sociales, arts et sciences humaines, de l'Universidad Nacional Abierta y a Distancia -UNAD.

** Ph.D. Théorie de l'Education et de pédagogie sociale. Professeur-chercheur. Universidad Nacional Abierta y a Distancia -UNAD. Adresse: Calle 14 Sur No. 14-23, Bogotá, Colombia. Email: julio.rojas@unad.edu.co.

*** M.Sc. Technologies d'Information appliquées à l'Education. Professeur auxiliare, Universidad Pedagógica Nacional UPN. Adresse: Calle 14 Sur No. 14-23, Bogotá, Colombia. Email: lalealu@pedagogica.edu.co. 


\section{Introducción}

La articulación de discursos, prácticas y ambientes en los aspectos relacionados con las tecnologías de la información y la comunicación (TIC) no ha sido fácil para las Instituciones de Educación Superior (IES) colombianas. La explicación más recurrente ofrecida para este fenómeno termina por circunscribirlo en el esquema de la confrontación de contrarios, en el que una de las partes en contienda es acusada de ser obsoleta, dogmática o de estar equivocada (tecnófobos/ tecnófilos, nativos/migrantes digitales). Sin embargo, el tiempo transcurre y el modelo explicativo ha servido, más bien, para generar señalamientos y exclusión de aquellos que disienten de los esquemas impuestos sin reflexión colectiva, ni consulta o participación académica.

Estas experiencias se han expresado y desarrollado en el mundo educativo a partir de dos corrientes, principalmente: por un lado, la educación a distancia virtual, y por el otro, la educación virtual entendida como informática educativa, adscrita como escenario de apoyo didáctico y administrativo en la tradicional educación presencial. Junto a estas corrientes, la semipresencialidad y el aprendizaje combinado, o b-learning, han habitado de manera directa en algunos casos $\mathrm{y}$ tangencial en otros. Con esto, han generado un escenario fronterizo que ha abierto discusiones y debates sobre temas relevantes de la educación contemporánea: la distancia y la presencia, el claustro y la desescolarización, lo físico y lo digital, el sentido general de lo virtual, etc. Indudablemente, el problema está ahí para ser estudiado y analizado desde diferentes perspectivas, en particular las de carácter interdisciplinario.

\section{Los sistemas culturales; un enfoque orientado al análisis de la universidad contemporánea}

El trabajo de esta investigación partió de la premisa de que la universidad actual está, efectivamente, muy lejos de poder ser analizada como una institución moderna con una identidad y un proyecto autónomo y unificado; o como una entidad que considera los procesos marginales, diferentes o antagónicos a ella como expresiones de resistencia a la institucionalidad o de resistencias al cambio. Por el contrario, parece más viable estudiarla como un sistema abierto, múltiple, pluridentitario y en permanente interacción y transformación.

Lo anterior permite registrar cómo la universidad alterna discursos que penden entre el arraigo a una identidad histórica y a una capacidad de permanente actualización. El principio de una identidad sujeta a la tradición se juega en cada cambio que desborda el control institucional, debido a la intervención de diferentes actores con diferentes intereses (Rojas, 2012). 
Para el caso de este estudio, lo anterior es visible en las tres universidades seleccionadas: Universidad Nacional Abierta y a Distancia (UNAD), Universidad Santo Tomás (USTA) y Universidad Pedagógica Nacional (UPN).

Para advertir la apuesta por la identidad, en el caso de la UNAD, el problema de la identidad institucional es puesto en cuestión a partir de la invocación de su misión:

El macro-problema formulado indica que la UNAD (antigua UNISUR) no ha desarrollado plenamente su identidad como Universidad Abierta y a Distancia, constituida intencionalmente para servir a las comunidades regionales, carentes de oportunidades educativas, mediante medios, mediadores y mediaciones pedagógicas, y el uso didáctico y sistemático de las TIC, en interacción con los contextos socioculturales y productivos, para la formación integral de los estudiantes, a partir del aprendizaje autónomo (UNAD, 2006, p. 19)

Este juego de representaciones simbólicas de identidad también hace presencia en la UPN, cuya identidad institucional se constituye con base en tres aspectos: la epistemologización pedagógica, el empoderamiento del acto educativo y pedagógico, y el reto de ofrecer los elementos pedagógicos fundamentales para la estructuración e implementación de la política educativa pública.

El esquema de conocimiento es secuencial, histórico y con una pesada carga de tradición basada en una identidad histórica: "A diferencia de nuestra estructura biológica, que se reproduce por información genética, el orden de la cultura y la sociedad lo hace por medio de procesos educativos. Esta es la vía por la cual cada nueva generación hereda ese enorme y diverso capital cultural de la humanidad, conformado durante milenios" (UPN, 2006, p. 52).

Este hecho enunciativo se reproduce de manera sistemática y similar en la USTA, aunque no desde una perspectiva republicana y liberal como en el caso de la UPN, sino más bien desde una perspectiva teológica y conservadora:

Respecto de la naturaleza de la universidad, los adjetivos de "católica" y "tomasino" no son agregados accidentalmente a su identidad y razón de ser originaria. La universalidad del saber solo es posible en la medida en que se incluya la racionalidad filosófica y teológica; hacia ello apuntan el humanismo integral y la formación integral. La USTA, por tanto, apela a su propia e inmanente forma filosófica y, desde allí, da sentido a las diversas formas del saber. (USTA, 2004, p. 131)

El discurso de la tradición se plantea entonces como un referente fundamental para la conservación del control. Las formaciones de identidades no están exentas de poder; cada concepto, cada enunciado, está puesto allí para mantener el control de un ordenamiento del mundo delimitado por intereses específicos y 
hegemónicos del sistema cultural y en conexión con formaciones discursivas exógenas insertadas en el flujo de estos intereses.

Dichos intereses han globalizado históricamente sentidos culturales al punto de generar fenómenos mundiales de racismo, de xenofobia, y de discriminación social y de género, sexo, edad y estatus económico. La tradición permite elevar juicios de valor sobre la diferencia emergente amparados en el valor de la verdad académica e investigativa:

Este hombre atemporal, ya no da cuenta del pasado, de su arraigo, no desea sentirse apegado a nada, parece de mal gusto, poco cool siquiera mencionarlo. La mundialización y el consumo desdibujaron la riqueza que cohabitaba. El pasado, los lazos de cultura. En el ciberespacio se pertenece a un hipermundo, sin fronteras, sin rasgos, allí se está; los individuos se integran al hipermundo, a los mundos, como participantes, como avatares. En estos mundos, en el hipermundo, no importan las raíces, la religión, el linaje familiar, o la posición política o económica. El avatar no da cuenta de su pasado, solo es, en el hoy, en el actual día. (Carreño et al., 2009, p. 28)

Ahora bien, el planteamiento discursivo relacionado con una renovación de la condición y de la naturaleza de la universidad establece una fricción entre el concepto tradicional de identidad: un lugar estático, trascendente y metafísico materializado en el claustro académico físico y tradicional, y por el contrario: una noción de virtualización de la realidad que involucra de manera directa e incisiva las TIC y los ambientes digitales en la vida universitaria.

De esta manera, la universidad ve emerger nuevas prácticas discursivas que presagian no el cambio teleológico y evolutivo de su tradición, sino la disrupción en referencia a una alteridad que se impone por segmentos y dibuja sus inconsistencias y sus discontinuidades históricas y metafísicas, develando la multiplicidad en su interior. La identidad pierde su naturaleza ontológica y esencialista, para ser leída de manera plural en la práctica académica e investigativa. El pasado, presentado por esta última como homogéneo, bucólico y tranquilizador, es leído por un "otro" advenedizo, sin estatus ni categoría moral, ontológica o epistemológica, que confronta su aparente coherencia, racionalidad y hegemonía.

El "otro" desnuda un pasado desordenado y atiborrado de saltos mutantes, ejercicios de control y neutralización de diferencias nocivas para el sistema de poder. En un efecto espejo, se lo presenta como síntesis de su condición histórica. Frente a ello, la tradición presenta su insatisfacción:

Solo quedan las ofertas tranquilizadoras, anestésicas, bellas y ante todo atemporales. No debe preocupar el tiempo como linealidad, como continuidad, es chocante, ofensiva, pues remite al fracaso del pasado y a la cruel incertidumbre del presente. Los múltiples tiempos posibles ofrecen una gama halagadora y optimista de 
los anhelos del hoy, sin fecha de expiración (Carreño et al., 2009, pp. 27-28)

La investigación del profesor S. Weisneir (2004), realizada con sus estudiantes del Programa de Comunicación de la USTA, devela el carácter múltiple y local de la universidad. Abre una brecha en la perspectiva tradicional de la comunicación en la universidad y en su manera de entender la identidad y la cultura.

El trabajo hace énfasis en el sentido cultural que constituye a las comunidades, y en cómo en todo sistema cultural las diferencias son múltiples y se crean escenarios y redes para que estas circulen estableciendo todo tipo de relaciones complementarias, de oposición, de contenencia e incluso de confrontación ideológica, como lo plantea Weisneir.

Las identidades son múltiples, se construyen en diversos contextos de alteridad y se reproducen en diferentes ambientes físicos y digitales, y muchas veces en ambos simultáneamente. Las características de los ambientes imprimen sellos específicos al desarrollo de dichas identidades y permiten empoderarlas incluso en escenarios políticos:

Nos interesamos en la innovación en relación con los procesos de construcción de identidades en las narraciones. En efecto, consideramos que, desde una perspectiva dinámica de las identidades, las narrativas puestas en juego en los "sitios web", y generadas a propósito de estos, son lugares de construcción y expresión de procesos de reconocimiento y desconocimiento constante que hacen visible la formulación, la revisión o el cambio de esquemas de construcción de la realidad y de los individuos o grupos dentro de ellos (Weisneir, 2004, p. 51).

\section{Tensiones y transiciones}

En dicho contexto, la institucionalidad universitaria se juega el cambio en la dirección de su enunciado normativo.

En la USTA, se inserta el concepto de cibercultura como indicador del cambio en la sociedad académica global, y a partir del 2004, inaugura sus primeros procesos para la conformación de un Comité de Educación Virtual:

Desde la perspectiva de su proyecto educativo universitario, la Universidad Santo Tomás se juega la explicación en el cambio de rumbo: asistimos hoy a otro reto en el campo educativo y cultural: el avance y la penetración del mundo audiovisual (cine, radio, televisión) que promueve un nuevo imaginario y un nuevo estilo de comunicación. De ahí que la implementación de un sistema mundial complejo de comunicación electrónica está cambiando 
rápidamente nuestros patrones tradicionales de cultura, ante el impacto de los medios de comunicación de masas (...) hasta llegar al medio más usual de comunicación y de procesamiento, en los 90, de la información (internet), que de estrategia militar se ha convertido en el motor más notorio de los nuevos cambios que nos introducen a un nuevo ámbito: la cibercultura. (USTA, 2009, p. 40)

Este mismo escenario es identificado con otra metáfora: "la revolución digital", que plantea una necesaria tradición en dicha revolución:

Dicho reto en la USTA viene siendo asumido desde hace décadas con los sistemas de enseñanza a distancia VUAD, que diseña sus procesos pedagógicos con innovaciones tecnológicas y con un nuevo currículo, que se adapta al principio de lo virtual y que asume en los programas presenciales una política de complemento necesario a las metodologías igualmente presenciales (USTA, 2009, p. 40)

Por su parte, la UNAD analiza el evento como una oportunidad para insertarse en los ambientes digitales y distanciarse de la práctica semipresencial tradicional. A partir de allí, promulga un nuevo origen basado en el reencauzamiento del principio, emprende una agresiva política de migración de más del $70 \%$ de su oferta educativa hacia los ambientes digitales y amplía su cobertura educativa en número de estudiantes de 23.739 en el 2004 a 63.108 en el 2012 (ECSAH-UNAD, 2012). "La capacidad de aprender y de auto-regularse les permite a las organizaciones auto-desarrollarse y crecer sin perder su identidad, e igualmente reconocer el valor pedagógico e histórico de las organizaciones" (UNAD, 2006, p. 2).

La gran pregunta es: ¿cuál identidad se mantiene estática en procesos de gran transformación? Sin duda, esto plantea la primera tensión, expresada en el acontecimiento administrativo, de transformación entre el discurso de la institución moderna y el de la organización contemporánea. Mientras la primera se sostiene sobre una idea de identidad inmutable en medio de la dispersión exógena a su sistema, la segunda aborda la interacción y la relación entre los diferentes nodos de su sistema como objeto e impulso de sus transformaciones de identidad.

Dicha transformación se presenta como aceptación de nuevas prácticas y nuevos sujetos en la UPN (2006, p. 89): “La velocidad del conocimiento en la sociedad contemporánea y las formas de acceder a él han generado dinámicas diferentes de enseñanza y aprendizaje, nuevas formas y ritmos del conocer, nuevas concepciones de sujeto y de subjetividad". Junto a ello, el sector administrativo comienza a desarrollar sus enunciados para promover una iniciativa de e-learning que permita justificar la incursión de la modalidad dentro de la oferta universitaria (UPN, 2004).

En este contexto, surge el Instituto de Tecnologías Abiertas en Educación (ITAE), como alternativa a una perspectiva de identidad uniformada en la presencialidad, sostenida como modalidad educativa exclusiva por la UPN durante más de medio siglo. 
Había una consideración inicial a partir de lo que podría ser el eje que ha movido todo este asunto, no solo en la UPN sino en las diferentes instituciones de educación superior, es el eje de las Tecnologías de Información y Comunicación. (Rozo, comunicación personal, julio 28 de 2008)

Todo este movimiento se traduce en la incorporación de categorías que no son nuevas pero que no habían sido utilizadas antes por estas universidades. Entre otras, se encuentran: megauniversidad (Daniel, 2000; Rama, 2011; UNAD, 2007), e-learning (UPN, 2006), tecnocultura, sociedades del conocimiento, Ambientes Virtuales de Aprendizaje, reticularidad, heterarquía, fractalidad (Secretaría General UNAD, 2004; UNAD, 2007), revolución digital (USTA, 2004; USTA, 2009), Tecnologías Abiertas en Educación (Rozo, 2010), entre otros más.

\section{Hacia una concepción de sistema cultural universitario}

Aunque a primera vista pareciera que el sistema cultural universitario orienta y determina los discursos, las prácticas y los ambientes surgidos en relación con lo digital, la verdad es que estos desbordan completamente el control realizado por parte del sistema universitario, en razón de criterios de multiplicidad, alteridad y pluridentidad. Finalmente, terminan develando una reagrupación de hechos y experiencias en el tiempo de dicha universidad, enmarcadas no en la noción tradicional de historia, sino en el concepto de evento (Foucault, 1970; Sánchez, 2010).

Llevado este esquema explicativo al ámbito de los ambientes virtuales, se puede decir que su carácter fronterizo los hace permanecer aparentemente inmóviles o con una dinámica excesivamente lenta en algunos sistemas, como en la UPN o en la USTA, por ejemplo, mientras que en otros sistemas, como en la UNAD, cuentan con protagonismo y dinamismo intensos. Por sus diferencias de ritmo y por sus características de uso, las TIC obligan a estos sistemas universitarios a pensar, como nunca antes, en su alteridad de manera consciente y permanente.

Con el análisis adelantado, se pudo apreciar cómo la diversidad existente en el interior de la universidad, en sus distintas expresiones, es positiva y al final la enriquece. Dependiendo de los ambientes que se creen para permitir o negar dicha diversidad, la universidad avanza o se paraliza en sus procesos académicos y administrativos.

El trabajo se abordó a partir del esquema explicativo de los "sistemas culturales" entendidos como escenarios de multiplicidad, pluridentidad y alteridad en las sociedades humanas. Este movimiento de interiorización-exteriorización dio lugar a identificar dispositivos de dinamización de dichos escenarios: mito/ discurso, rito/práctica y territorio/contexto, que provocan su transformación 
permanente y con ello, la necesidad de actualizar de manera constante su lectura y comprensión.

En este estudio, nos referimos a la manera como la universidad se revitaliza continuamente de acuerdo con el diálogo interdisciplinar, intercultural, inter y transdisciplinar en contextos locales y globales a escala.

La aplicación del enfoque teórico propuesto permitió analizar la realidad universitaria como un componente nodal y reticular de una multiplicidad cultural más amplia y sistémica, con la que se encuentra en permanente interacción y con la cual se transforma de manera igualmente múltiple y desigual.

El siguiente esquema expresa gráficamente esta doble condición en el sistema, en relación con su alteridad.

Figura 1. Noción gráfica de los sistemas culturales

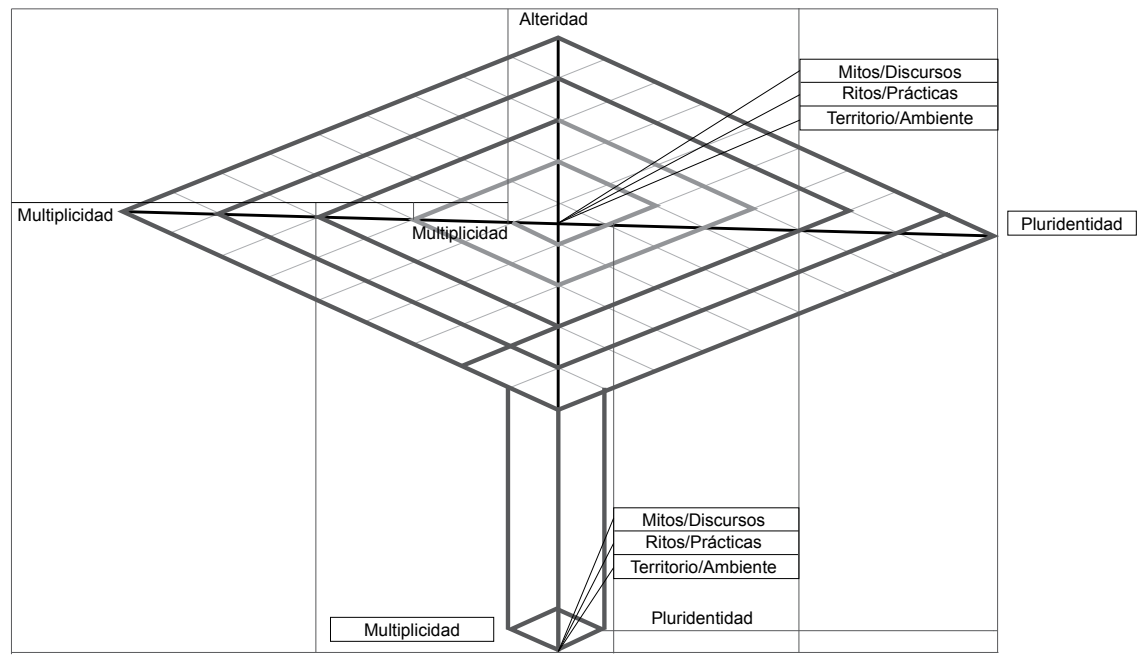

Fuente: elaboración propia.

\section{Del paradigma de sociedades configuradas como comunidades, hacia el paradigma de las sociedades representadas como redes: la era de la cibercultura}

El concepto de comunidad con el cual se cerró el siglo XX estaba íntimamente ligado al pensamiento positivista y moderno de los últimos siglos. Desde las descripciones sobre el feudalismo y la identidad asociada con la tierra, la cultura y la tradición de los siglos XIII al XVII, hasta los múltiples e intensos esfuerzos 
de la antropología en los últimos tres siglos por definir el concepto a partir de los estudios en grupos étnicos, que igualmente asociaban comunidad con identidad, tradición y continuidad en el territorio geográfico: "por eso su discurso comunitario es perpetuación de la tradición; la tradición explica la comunidad, o mejor aún, la tradición es la comunidad" (Neira, 2005, p. 36).

Si bien no todos los actos de interacción social se pueden definir como comunitarios, mucha de la literatura de análisis social clásica, y la primera de finales del siglo XX, interpretó una gran mayoría de los fenómenos sociales sobre el soporte conceptual de la comunidad en todos los ámbitos y niveles (comunidad económica, académica, social, étnica, de base, etc.). Muchos de los trabajos y las investigaciones de finales del último siglo se desarrollaron según una lógica transicional.

McLuhan y Powers (1990) definieron el concepto de "aldea global" bajo dos ideas centrales: en primer lugar, la noción de comunidad trabajada tradicionalmente en las ciencias sociales en la relación cultura-territorio y tradición mencionada antes, y en segundo lugar, la perspectiva de mundo permanentemente conectado, vislumbrado en los intensos cambios sociales y culturales debido a lo que, en su momento, se denominó "revolución de las telecomunicaciones".

Castells (2002) retomó esa idea y la interpretó a la luz de los intensos y radicales cambios de finales del siglo XX y principios del XXI, para definir la sociedad contemporánea como una "sociedad red" conformada por pequeñas comunidades conectadas que ejercen una relación de asociación y disociación con el ámbito global: "En un mundo como este de cambio incontrolado y confuso, la gente tiende a reagruparse en torno a identidades primarias: religiosa, étnica, territorial, nacional" (Castells, 2002, p. 29).

Sin embargo, lo que se puede analizar de los estudios mencionados y otras perspectivas como la filosofía, la psicología, la sociología, la ciencia política, la economía, es que la crisis de la forma de concebir la sociedad y la cultura denunciada por la antropología en el último siglo, tenía eco en una profunda crisis filosófica de representación sobre la concepción del hombre y de sus producciones simbólicas (Beorlegui, 2004)

Lo anterior también se replicó en las crisis de método y de objeto que sostenía el aparato científico de conocimiento en las ciencias sociales y humanas (Foucault, 1971; Lyotard, 1987; Velasco y Díaz de Rada, 1997). Dichas crisis orientaron un giro en la perspectiva del mundo hacia construcciones de multiplicidad y diferencia tanto fuera como dentro de lo que tradicionalmente se había concebido como comunidad e identidad (Adler-Lomnitz, 2012).

En consecuencia de ello, los estudios sobre redes sociales que se venían trabajando desde mediados del siglo XX desde la psicología, la antropología, la sociología, la biología y la matemática, comenzaron a visibilizarse y a protagonizar una forma de lectura y análisis de las sociedades del siglo XXI. 
Las redes sociales se pueden entender como grupos de sujetos individuales-colectivos, vinculados entre sí a través de una relación o un conjunto de relaciones sociales: "La idea central del análisis de redes reside en el supuesto de que lo que la gente siente, piensa y hace tiene su origen y se manifiesta en las pautas de las relaciones que se dan entre actores" (Lozares, 1996, p. 110). Este hecho relativiza, en el análisis central, categorías como raza, sexo o situación social, y redefine los lugares a partir de los cuales se pueden comprender y explicar nociones como sociedad, identidad, cultura, aprendizaje, movilidad, inclusión etc.

Los estudios referentes al concepto de redes sociales se visibilizan popularmente con el concepto de sociedad red trabajado en los noventa (Castells, 2002). No obstante, era un tema que venía siendo trabajado desde principios del siglo XX: a partir de la psicología, con el trabajo de la Gestalt y del enfoque de la "sociometría"; de la antropología con el estructuralismo; de la matemática con la teoría de grafos (Lozares, 1996).

En la actualidad, el tema cobra gran importancia y se consolida con la articulación de este enfoque en los actuales escenarios de virtualización de la sociedad, la cultura, la economía, la política, el territorio, las comunicaciones, las interacciones sociales y el arte, entre otros. Los trabajos de Watts acerca de su teoría de "redes de pequeño mundo" (Duncat y Strogatz, 1998) enriquecieron profundamente el estudio sobre redes en internet, al cual se han sumado los trabajos de Barabasi (2002) -del Machassusets Institute of Technology (MIT)-, con su enfoque sobre redes de libre agregación, y de Urrutia (2001), con sus estudios sobre índices de rebeldía en las redes sociales; en las ciencias sociales y educativas, han propiciado múltiples investigaciones sobre la relación entre educación y redes sociales (Dominguez y Álvarez, 2012; Guerrero, 2010; Rojas y Salazar, 2012)

Al respecto, en el estudio presentado aquí, algunas de las reflexiones docentes en torno a la relación en ambientes virtuales registran una transformación de la percepción de mundo, de identidad y de cultura tanto en el sujeto individual como colectivo. El hecho se puede analizar desde diferentes situaciones:

En primer lugar, un sentido de recreación identitaria producto de la interacción con sujetos procedentes de diferentes sistemas culturales:

Cuando te relacionas con otra persona en la virtualidad y además sabes que esa persona no es de tu contexto, tú procuras de alguna forma reflexionar antes de escribir sobre lo que le vas a decir y esa reflexión de alguna forma implica, pienso, implica una reflexión también sobre tu propia identidad. Entonces, en algunos casos dicen que eso es simulación, pero yo creo que de alguna forma esa simulación termina afectando la relación cotidiana que tú tienes con los otros con los que convives, termina afectando tu convivencia. Entonces, en esa medida, considero que es una buena manera de darle cierta resignificación a la manera como nos relacionamos en la cotidianidad física. (Iris Gómez, comunicación personal, noviembre 20 de 2007) 
En segundo lugar, un sentido de virtualidad expuesto como interacción y encuentro por encima de otras categorías como la espacialidad. Dicha reflexión proporciona elementos ontológicos para el análisis del hecho educativo. Este aspecto se puede observar en el siguiente caso; los profesores Luis Facundo Maldonado de la UPN y Roberto Salazar de la UNAD, en reuniones conjuntas sobre el tema, sostienen percepciones diferentes acerca del problema de la interacción y la educación a distancia. Finalmente, el profesor Maldonado saca una conclusión recogida en dicha experiencia y que es muy interesante en este trabajo:

\begin{abstract}
Ahora, el fenómeno está en que el maestro perdió poder cuando se fue el artefacto y entonces estamos ante el fenómeno de educación a distancia. Este fenómeno del aprendizaje enredado en la educación autónoma. A mí, individualmente, el fenómeno de educación a distancia no me puede ir, o sea, no me cautiva. Yo le decía a Roberto Salazar [vicerrector de Mediaciones Tecnopedagógicas de la UNAD] y a los que estaban ahí: "La educación a distancia... Uno está con artefactos acercándose, uno no se está alejando, entonces para qué poner como prevalente el concepto de la distancia si en realidad es el encuentro lo que está sucediendo" (Luis Facundo Maldonado, comunicación personal, noviembre 30 de 2007).
\end{abstract}

En tercer lugar, el fenómeno de reticularidad desnuda la intrincada red de intereses en la cual fluyen los sujetos que fortalecen o cuestionan prácticas discursivas existentes o en emergencia. En muchos casos, este contacto entre un afuera y un adentro de la práctica discursiva del sujeto ni siquiera está exclusivamente determinado por el sujeto mismo, sino también, en gran medida, por la alteridad que lo rodea en diferentes grados y niveles, y que lo atraviesa como un vector de poder, buscando construir subjetividad en él.

Muchos docentes representan la expresión de una forma ontológica de saberpoder asentada en una forma cosmogónica de la presencialidad. Se identifica en la semipresencialidad de la EAD en la UNAD y en la USTA, así como en la presencialidad de la UPN. Y sin embargo, a pesar de la fuerza de la tradición y de una, todavía fuerte, legitimidad de esta forma determinista de la presencialidad, la emergencia de nuevas prácticas discursivas irrumpe con violencia epistémica y metodológica desgarrando el discurso, la práctica y los ambientes asentados allí en el sujeto. En este caso, el desgarramiento se desarrolla sobre el cuestionamiento ontológico a una interpretación exclusiva de la presencialidad:

Se han generado otros elementos más ideológicos como las teorías de la alienación. El medio aliena, y como el medio aliena la única perspectiva de salvación es la enseñanza directa, que es liberadora. Todo un imaginario ideologizado de la relación directa, en donde la palabra del maestro es la palabra, la mirada del maestro. Lo que yo llamaría en un sentido la pedagogía de los hedores y los 
humores. Si te coloco en mi axila, entonces, esa colocación opera en términos mecánicos como ese proceso humanizado de formación. Esos procesos puede observarlos uno todavía en la UNAD, a pesar de que es una institución de educación a distancia, en muchos de sus docentes y tutores (Roberto Salazar, vicerrector de Medios y Mediaciones de la UNAD, comunicación personal, junio 5 de 2009).

En cuarto lugar, se puede hablar de una diferencia entre la concepción acerca de lo virtual en el desarrollo de la propuesta universitaria y la práctica discursiva relacionada con la construcción de valores entre lo físico y lo digital.

Por un lado, se concibe el campus virtual como un reducido espacio digital: en la USTA y en la UNAD se restringe al escenario construido por el administrador de contenidos educativos, que para el caso es la plataforma Moodle. En la UPN, el concepto es un poco más amplio y abarca la página web institucional de la universidad.

Por el otro lado, se conciben los ambientes digitales como una continuación de la realidad física, como un escenario en donde la gente se juega las mismas pasiones que en la vida física y sobre el que hay que aprender a transitar. En Facebook o en hi5 hay unos grupos de interés que cuando tú te pones a revisarlos y a analizarlos dices: esta sociedad se está desintegrando, qué es esta locura, cómo es posible que tantas personas estén en contra de una persona, cómo es posible que tantas personas admiren a una sola persona. Pero eso es reflejo de lo que hay en la sociedad real. Lo que pasa es que allí, considero, hay una observación de esos sentimientos y esas emociones, porque tu identidad puede ser cambiada, puede ser desdibujada, tú puedes ser la amiga de una niña de 14 años y puedes ser fácilmente un hombre de 60, pero eso que es el reflejo de una sociedad que está enferma se ve en la red. Al respecto, son reveladoras las reflexiones de Claudia Rozo, directora del Instituto de Tecnologías Abiertas en Educación (IATE) de la UPN (comunicación personal, agosto 7 de 2008).

Para Quiñones (2005), independientemente de las valoraciones éticas y morales, la sociedad ya se encuentra inmersa en nuevas construcciones que apuntan a una sociedad glocalizada, caracterizada por la multiplicidad de sus discursos, prácticas y ambientes, enfocada no en el sentido comunitario y tradicional de la relación historia-identidad, sino en la perspectiva reticular de la relación interacción-red-identidad.

\section{Conclusiones}

La entrada de las TIC, indudablemente, marca una ruptura discursiva en la manera como se entiende la construcción educativa, el reconocimiento de las formas en que se expresa su práctica y los ambientes que genera el concebir 
el mundo académico como un mundo virtual, en términos de las múltiples permutaciones que provoca la combinación de ambientes físicos y digitales.

Es complejo pensar el tema, y sobre todo, pensarlo en el cuerpo mismo del sistema cultural universitario. La universidad, acostumbrada al paradigma moderno de la objetividad y el distanciamiento de su objeto de estudio, se ve enfrentada a la urgente necesidad de pensar el fenómeno conforme sucede en su propia piel.

Hablar del aprendizaje y la educación significa, entonces, hablar de identidades -con artefactos, instrumentos, procesos y discursos relacionados con el ambiente tecnológico-. Hablar del individuo contemporáneo significa hablar, a su vez, de sus formas de virtualización, materializadas especialmente en las TIC, inseparables del ser humano en un contexto en donde la técnica y el artefacto son tan antiguos como su posibilidad de virtualización del mundo humano.

\section{$\mathbf{R}_{\text {eferencias }}$}

Adler-Lomnitz, L. (2012). Redes sociales, cultura y poder. Ensayos de antropología latinoamericana. México D.F.: Flacso.

Auge, M. (1995). Hacia una antropología de los mundos contemporáneos. Barcelona: Gedisa.

Barabasi, A. L. (2002). Linked: The new science of networks. Nueva York: Perseus Books Group.

Beorlegui, C. (2004). Antropología filosófica: nosotros urdimbre solidaria y responsable (2. ${ }^{\text {a }}$ ed.). Bilbao: Universidad de Deusto.

Carreño, D., Restrepo, A., Rojas, A. y Valero, H. (2009). Reflexión filosófica hacia la construcción de un modelo humanista de educación virtual. Informe de investigación. Departamento de Humanidades. Grupo de Investigación social y humanística. Bogotá: USTA.

Castells, M. (2002). La era de la información. Vol. I: la sociedad red. México D.F.: Siglo XXI.

Centro de Investigación de las Telecomunicaciones (CINTEL). (2008). Brecha digital en Colombia. Bogotá: Autor.

Daniel, J. (2000). El surgimiento de las megauniversidades. En Leer, A. (ed.). La visión de los líderes en la era digital (pp. 333-342). México D.F.: Prentice Hall.

Dominguez, D. y Álvarez, J. F. (2012). Redes sociales y espacios universitarios. Conocimiento e innovación abierta en el espacio iberoamericano del 
conocimiento. Revista de Universidad y Sociedad del Conocimiento (RUSC), 9(1), 51-64. Recuperado el 28 de febrero de 2012, de http://rusc.uoc.edu/ojs/index. php/rusc/article/view/v9n1-dominguez-alvarez/v9n1-dominguezalvarez

Duncat, W. y Strogatz, S. (1998). Collective dynamics of «small-world» networks. Nature, 393, 440-442.

Escuela de Ciencias Sociales, Artes y Humanidades de la UNAD (ECSAHUNAD). (2012). Proyecto educativo de escuela. Bogotá: UNAD.

Facundo, A. (2009). Análisis sobre la deserción en la educación superior virtual en Colombia: el caso de la UNAD. Revista de Investigaciones UNAD, 8(2), 117-149.

Foucault, M. (1970). La arqueología del saber. Madrid: Siglo XXI.

Foucault, M. (1971). Las palabras y las cosas: Una arqueología de las ciencias humanas. México D.F.: Siglo XXI.

Gere, C. (2010). Algunas reflexiones sobre la cultura digital. En Alsina, P. (coord.). De la digitalización de la cultura a la cultura digital. Digithum, (12). Recuperado el 8 de agosto de 2013, de http://digithum.uoc.edu/ojs/ index.php/digithum/article/view/n12-gere/n12-gere-esp

Guerrero Suárez, C. (2010). La formación en red como objeto de estudio. Revista de Universidad y Sociedad del Conocimiento (RUSC), 7(2). Recuperado de http:// dialnet.unirioja.es/servlet/articulo?codigo $=3664428$

Hine, C. (2007). Multi-sited ethnography as a middle range methodology for contemporary STS. Science, Technology and Human Values, 2(42).

Huberman, L. (2005). Los bienes terrenales del hombre: historia de las riquezas de las naciones. Bogotá: Génesis.

Leal, A. J. (2007). Normativa institucional UNAD. Presentación. Bogotá: UNAD.

Lozares, C. (1996). La teoría de redes sociales. Papers, Revista de Sociología, Universidad de Barcelona, (48), 103-126.

Lyotard, J. F. (1987). La condición posmoderna: informe sobre el saber (M. Antolín Rato, trad.). Madrid: Cátedra.

McLuhan, M. y Powers, B. (1990). La aldea global. Barcelona: Gedisa.

Neira, Y. C. (2005). Teoría transnacional: revisitando la comunidad de los antropólogos. Política y Cultura, (23), 181-194. Recuperado de http://www. redalyc.org/pdf/267/26702311.pdf

Quiñones, F. (2005). De la cultura a la cibercultura. Revista Hallazgos, (4), 174-190. 
Rama, C. (2011). La educación superior en América Latina en el periodo 20002010: ocho ejes centrales en discusión. Revista Innovacíon Educativa, 11(57), 15-20.

Restrepo, E. (2008a). Cuestiones de método: “eventualización” y problematización en Foucault. Tabula Rasa, (8), 111-132.

Restrepo, E. (2008b). Multiculturalismo, gubernamentalidad, resistencia. El giro hermenéutico de las ciencias sociales y humanas. En García, Ó. A. El giro hermenéutico de las ciencias sociales y humanas: diálogo con la sociología (pp. 35-48). Medellín: Universidad Nacional de Colombia.

Rodriguez, J. A. (2008). El mundo virtual como dispositivo para la creación artística. Nómadas, (28), 138-147.

Rojas, M. J. y Salazar, M. J. (2012). Cibercultura: una forma contemporánea de comunicación multimedia. Análisis, (81), 167-191. Recuperado de http:// revistas.usta.edu.co/index.php/analisis/article/view/1276/1474

Rojas Mesa, J. E. (2012). Prácticas, ambientes y saberes: políticas de TIC en el contexto de la educación superior colombiana. Civilizar: Ciencias sociales y humanas. Revista de divulgación científica, (23), 169-189. Recuperado el 20 de agosto de 2013, de http://www.usergioarboleda.edu.co/civilizar/practicas_ambientes_y_saberes.htm

Rozo Sandoval, C. (2010). Dimensión pedagógica de la educación virtual: una reflexión pendiente. Pedagogía y saberes, (32), 33-44.

Rueda, R. (2008). Cibercultura: metáforas, prácticas sociales y colectivos en red. Nómadas, (28), 8-20. Recuperado de http://www.ucentral.edu.co/images/ editorial/nomadas/docs/nomadas_1_cibercultura.pdf

Sánchez Amaya, T. (2010). La caja de herramientas como opción metodológica. Análisis, (76), 71-102.

Sanz Adrados, J. J. (2006). Elementos para un marco conceptual sobre la incorporación de las TIC en educación. Cuadernos de Filosofía Latinoamericana, 27(94), 200-206.

Turkle, S. (2009). Simulation and its discontens. Cambridge: MIT Press.

Turkle, S. (2011). Alone together: Why we expect more from techonology and less from each other. Massachusets: Basic Books.

Universidad Nacional Abierta y a Distancia (UNAD). (2004). Normatividad institucional. Bogotá: Autor. . (2006). Acuerdo 001, 29 de agosto de 2006. Estatuto General UNAD. Secretaría General. Capítulo 1. Bogotá: Autor. 
. (2007). La megauniversidad: un concepto que toma fuerza en nuestra institución. Recuperado el 13 de diciembre de 2010, de http://www.unad.edu.co/boletin/ comunad/pages/boletin-marzo-1/la_megauniversidad.htm

Universidad Pedagógica Nacional (UPN). (2004). Boletin Estadístico 2000-2004. Bogotá: Autor.

. (2006). Proyecto político pedagógico de la Universidad Pedagógica Nacional. Proyecto Educativo Institucional. Bogotá: Autor.

Urrutia, J. (2001). Redes de personas, internet y la lógica de la abundancia. Un paseo por la Nueva Economía. Ekonomiaz, 46, 182-201.

Universidad Santo Tomás (USTA). (2004). Proyecto Educativo Institucional. Bogotá: Autor. . (2009). Modelo Educativo Pedagógico. Bogotá: Autor.

Velasco, H. y Díaz de Rada, A. (1997). La lógica de la investigación etnográfica: un modelo de trabajo para etnógrafos de escuela. Madrid: Trotta.

Weisneir, S. (2004). Internet, guerra y paz en Colombia: conflicto, narrativas e identidades. Hallazgos, 1(1), 50-57. 